\title{
AN ENHANCED SEEDS CATEGORIZATION AND CLASSIFICATION BASED ON MULTIPLE FEATURES-SET
}

\author{
Mohamed Ibrahim Habib \\ Computer Science Department, College of Computing and Informatics, Saudi Electronic University, Riyadh \\ 11673, Saudi Arabia, \\ Faculty of Engineering, Port Said University, Port Said, 42526 Egypt, \\ mhabib@seu.edu.sa
}

\begin{abstract}
Seed classification contributes significantly to the final added value in crop production. Manual seed characteristics estimation is a difficult, time-consuming process that is prone to human error. Image processing is a good candidate for developing automated seed characteristics estimation systems. Many applications use image processing, such as inspecting pests in crop fields, grading fruits by size and color, estimating ripeness of fruits and vegetables before harvesting, and identifying crop diseases. For the classification of different seed varieties, old methods are still used. Manual seed size calculation and classification (small, medium, and large) with calipers, for example. There are no state-of-the-art methods for classifying seeds within species. This study will look at various image processing techniques for estimating various seed characteristics, improving classification, discovering new features, reducing complexity, and identifying flaws in existing techniques. According to the findings, there is a negative relationship between soya bean seed size and climatic factors. The decrease in osmotic ability affects larger seeds because they need more water for growth. As a result, seed selection is important prior to plantation based on environmental conditions.
\end{abstract}

Keywords: Seed Classification, Feature Extraction, Machine Learning Classification, and Image Processing.

\section{Introduction}

There has been a lot of research done on various aspects of automated (computer vision based) agriculture systems (Liu et al., 2013; Varma \& Kanakadurga, 2013). The overall quality and rate of vegetative growth are influenced by seed characterization and classification. Smart Grain software (Tanabata et al., 2012) is proposed, which uses image processing to measure seed shape quickly and accurately. This method calculates seed parameters very quickly and accurately. However, essential characteristics of seeds such as color and texture are not considered by the process.

Seed size measurement from non-singulated samples and seed size distribution estimation are presented in (Shahin \& Symons, 2005). When compared to the manual sieving method, the image processing method provided more reliable and accurate performance. The process, however, does not allow for the removal of impurities from the bulk sample. Another research (Kumar et al., 2013) use image processing to estimate the shape-related geometric parameters and volume of five different types of dry beans. To measure and process the digital images, an image processing technique was used on two separate image orientations (longitudinal and lateral) of beans. The system is restricted to morphological characteristics, and image acquisition is vulnerable to errors due to digital camera height change.

The area calculation of seed from distorted images has been proposed in (A. Chaugule \& Mali, 2013) for quality seed selection. The acquisition of the image, the addition of noise to the image, and the implementation of various image quality metrics are the major steps. Regardless of noise distribution or intensity, the method calculates the precise seed area.

Seed size has a significant impact on crop behavior, and seed quality is often directly related to seed size. In (Mosavian \& Eshraghi-Nejad, 2013) three different cultivars (Mahdavi, Pishtaz and Bahar) of bread wheat are analyzed. Based on seed sizes, the characteristics were compared. The results show that seed size has no 
significant impact on germination percentage, but that smaller seed size has the highest germination rate; plants with larger seed sizes have higher seedling dry weight and grain yield than plants with smaller seed sizes.

Seed quality is determined by characteristics such as uniformity, color, and texture. Seed uniformity is observed since large size soybeans do not taste good and are used for oil production whereas smaller size soybeans are used for cooking.

Jinwei in (Li Jinwei et al., 2008) uses two approaches to recognize the color of a single rape seed: the common method and the major method. Image acquisition with a digital camera, image segmentation, color space transformation from RGB (red, green, blue) to HSV (hue, saturation, value) and NCM (nine color model), and color estimation using popular and significant methods are the main steps. When it came to determining the color of the seed, the main approach produced better results. Both methods, however, can only recognize pure yellow rapeseed and cannot reliably discern mixed yellow rapeseed, and color estimation can be inaccurate due to variations in illumination settings during image acquisition.

A method for counting clustered soybean seeds was proposed in (Kaur, 2014). The major steps of automated counting of seeds are image conversion from RGB to gray scale, then contrast limited adaptive histogram equalization, thresholding, dilation, masking, edge detection and counting with respect to shape. The algorithm offers a cost-effective and reliable solution. The overall accuracy, however, is dependent on the size of the soybean seed.

Good seeds help to germinate good agricultural products. However, identifying/classifying successful seeds prior to germination is extremely difficult. Pandey in (Pandey et al., 2013) proposes a content-based image retrieval technique for seed recognition. Artificial neural networks and the Euclidean distance method are used to automatically classify seeds based on shape and color features. The method's major drawbacks are that it needs single seed images for training (which is time consuming) and that the texture characteristics of the seed are not considered.

The use of artificial neural network classifiers to classify corn varieties based on mixed morphological and color features is proposed in (Pazoki et al., 2013). The neuro-fuzzy classifier outperformed MLP in terms of precision. It's worth noting that there are no texture features. The absence of texture features is noted. Multiple machine learning algorithms has been applied for the process of classification of corn seeds in (Ali et al., 2020). Random forest (RF), BayesNet (BN), LogitBoost (LB), and Multilayer Perceptron (MLP) were used to construct classification models using an optimized multi-feature (10-fold) cross-validation method. The MLP had a high level of classification accuracy $98.93 \%$.

A Comparative study of rice variety has been introduced in (Truong Hoang et al., 2020), an automation system for classifying rice seeds is developed using convolutional neural networks (CNN). The experiment yielded an important outcome. The DenNet21 framework yielded the highest accuracy of 99.04 percent. Using a combination of spatial and spectral features derived from high resolution RGB and hyperspectral images, an innovative technique for automatically screening and classifying rice seed is presented in (Fabiyi et al., 2020). The results show that by integrating spatial features extracted from high spatial resolution images with spectral features extracted from hyperspectral data cubes, the algorithm is successful in eliminating impure species.

A study of various machine vision techniques for seed quality assessment is carried out in (Belsare \& Dewasthale, 2013). The aim of this study is to develop imaging technology for monitoring seed imbibition (water absorption by dry seed), germination activity, and seed size and shape parameters. A study of various image processing applications in agriculture were introduced in (M. A. Chaugule, 2012). Different image processing techniques were investigated, as well as weed identification, parameter analysis, and fruit grading. The results of the parameter analysis show that image processing is reliable and timesaving, as well as providing better decisionmaking for agricultural purposes.

Seed characterization is a process that uses different parameters which can help us in differentiation and classification of different seeds, along with predictions on the yield of a specific crop. Basic features for seed characterization that are being used for research work are morphological, color and texture based. The area, perimeter, weight, and width of the seed are the general morphological characteristics. While Colors features represents properties of an image. Color identification in the RGB color space is inaccurate ("Comparison of Three Methods for Classification of Pizza Topping Using Different Color Space Transformations," 2005). For reliable color recognition, the HSV (hue, saturation, value) color space transformation is used. 
Texture features contains crucial details regarding a surface's structural arrangement. In (Haralick et al., 1973), textural feature for image classification has been presented, these features are estimated on the basis of Gray Level Co-Occurrence Matrix (GLCM). Also, in (Costa et al., 2012), algorithm for fractal analysis of texture is presented. Texture feature are extracted from the gray scale image using Segmentation based Fractal Textural Analysis (SFTA) algorithm.

Another major texture feature was introduced in (Amadasun \& King, 1989), the presented Neighborhood Gray Tone Difference Matrix (NGTDM) texture features have been defined as five different texture properties have been described: coarseness, contrast, busyness, complexity, and texture strength.

For the classification stage, a support vector machine (Boser et al., 1992; Cristianini \& Shawe-Taylor, 2000) is applied. The key benefits of SVM: it can obtain current possible solutions under finite samples; it can get global optimum solutions without falling into local optimums that traditional algorithms have; it converts nonlinear problems into linear problems by transforming them into a higher dimension space; and the complexity of the algorithm is unrelated to the dimension of space.

\section{Methodology}

Figure 1 shows the steps of proposed algorithm. The system will begin with capturing and collecting image samples for rice varieties. Then, a preprocessing phase to clean the captured images, followed by a feature extraction phase. A uniformity estimation is applied on the extracted features and a complete feature set will be built. As the number of features in the set is very large, a final step is added to select the most important features that affect the accuracy using support vector machine algorithm.

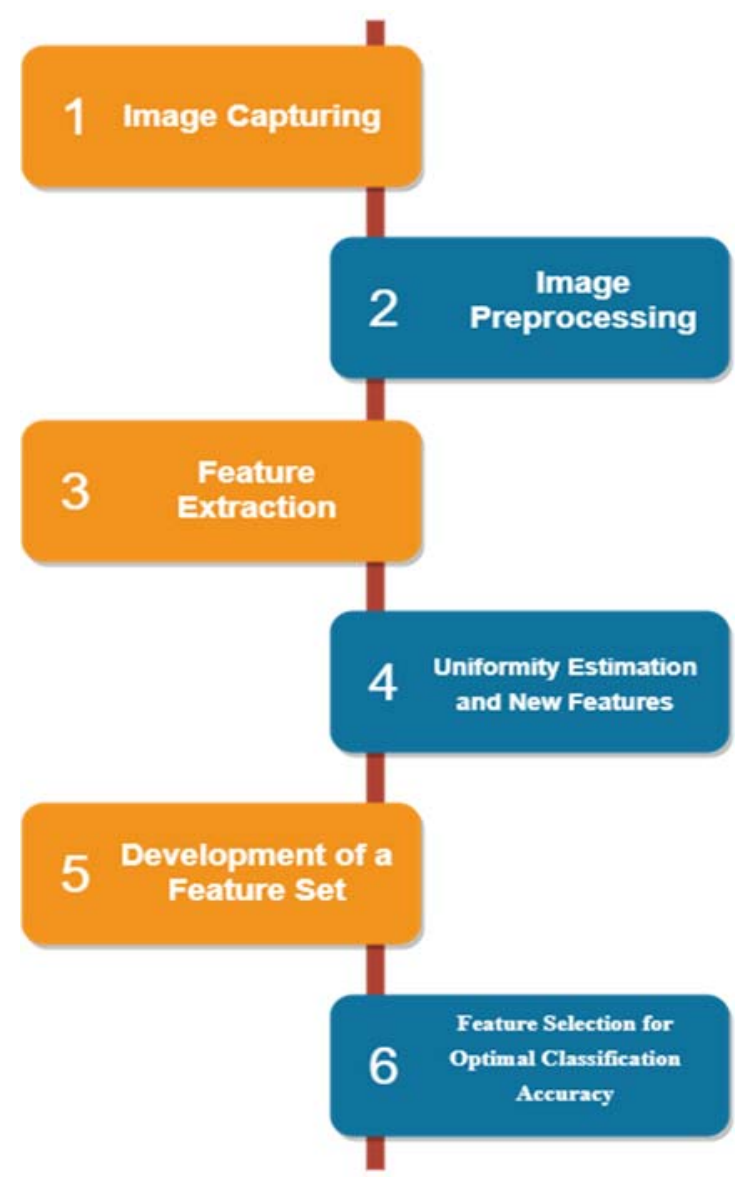

Fig. 1. Proposed Algorithm.

\subsection{Image Capturing}

The Fujitsu ScanSnap SV600 was used to capture the acquired samples of each variety at 600 dpi and 24bit color depth. Eight image sample of each variety and average total of $1200 \mathrm{kernel}$ of each variety were scanned. Each rice kernel was arranged in such a way that none of them would come into contact with one another. 


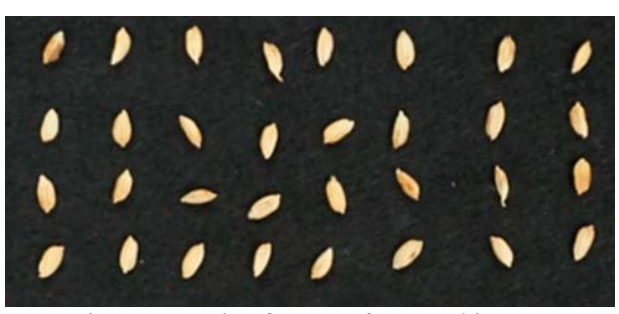

\subsection{Image Preprocessing}

In this phase, each scanned image pass through multi stages. Let the scanned image $\mathrm{S}$ with dimensions $\mathrm{L}$ and $\mathrm{W}$, where each image split into three channels $\mathrm{CH}=$ [red, green, blue]. Images are converted to gray scale image $\mathrm{G}$ using Eq. (1). Figure 3 represent a sample of generate gray scale image.

$$
G_{l \times w}=\left(\left(0.3 * S_{l \times w(C H=\text { red })}\right)+\left(0.59 * S_{l \times w(C H=\text { green })}\right)+\left(0.11 * S_{l \times w(C H=\text { blue })}\right)\right)
$$

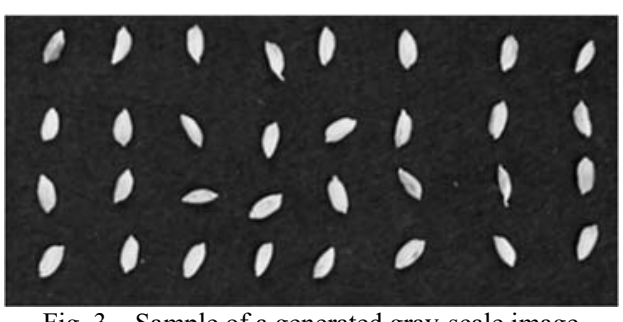

Fig. 3. Sample of a generated gray-scale image

Then the image is converted into binary. Where the threshold of the image has been computed based on the proposed method in (Liao et al., 2001). Figure 4 shows the generated binary image and Eq. (2) present the generation for pixels where Bin is the generated binary image, and $t$ the threshold.

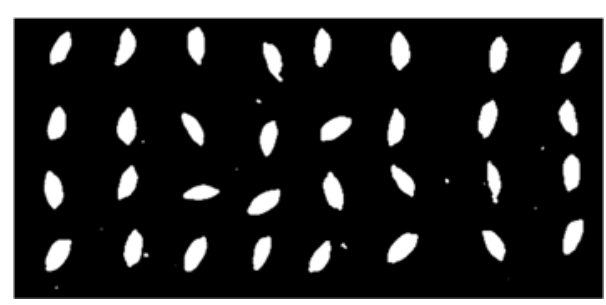

Fig. 4. Sample of a generated binary image

$$
\operatorname{Bin}_{l \times w}=\left\{\begin{array}{rll}
1, & \text { if } & G_{l \times w} \geq t \\
0, & \text { if } & G_{l \times w} \leq t
\end{array}\right.
$$

After this, the noise in the image that is caused by dust or broken fragments of rice kernels is removed. The average min. size of a single rice kernel is estimated as 4000 pixels, thus any opening with size less than 4000 pixel is blocked and the value of the pixels of that opening is set 0 .

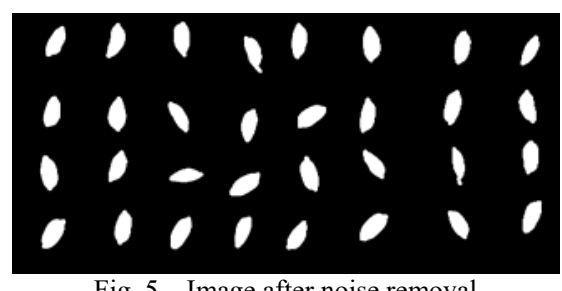

To distinguish each object in the binary image, linked component marking is used. A label matrix is created by labeling each object in the binary image. The photos below demonstrate how item marking is done. Let $\mathrm{Lm}$ refers to a labeled matrix with same size as generated binary image, $l$ denotes the label of object, $l$ starts from 1 to $n$ where $\mathrm{n}$ is the number of seeds in the image. 


$$
\begin{aligned}
& {\left[\begin{array}{cccccc}
1 & 1 & 0 & 0 & 1 & 1 \\
1 & 1 & 1 & 0 & 1 & 1 \\
1 & 1 & 1 & 0 & 0 & 1 \\
1 & 1 & 1 & 0 & 0 & 0 \\
1 & 1 & 1 & 0 & 0 & 1 \\
0 & 1 & 1 & 0 & 1 & 1
\end{array}\right]} \\
& \text { Fig. 6. } \\
& \text { Binary Image Sample }
\end{aligned}
$$

The seed image has been labeled in the same manner. Each individual seed's pixel is labeled as $1,2,3, \ldots$

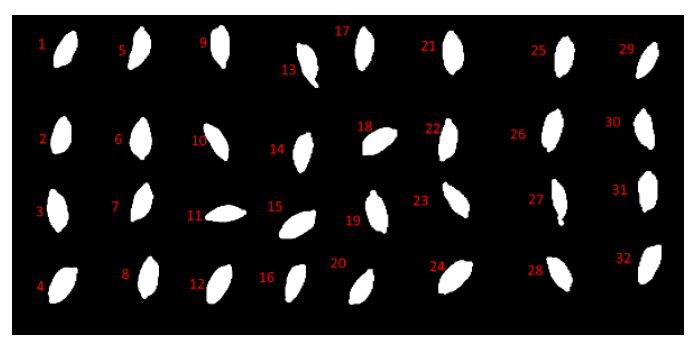

Fig. 8. Binary Image of labeled rice kernels

\subsection{Feature Extraction}

In the previous phase, separable objects in the binary image are attained. Then, three main categories of features are extracted as follows.

\subsubsection{Morphological Features}

Four main features are obtained, which are the perimeter, area, length, and width. To calculate the perimeter, Let $P_{i}$ be any pixels in the boundary of the object and total pixels number of pixels in the boundary of the seed be X, then perimeter $\mathrm{P}$ of the seed is equal to total number of pixel in the boundary of the object.

$$
P t=X
$$

The area of the seed is equal to the total number of pixels in the image. Let $N$ denotes the total number of pixels in an object then area $A$ of the seed is:

$$
A=N
$$

The Euclidean distance between the two farthest points on the perimeter $P i=(x i, y i)$ of a seed is equal to its length $L . H l(i, j)$ Be the distance between all pairs of points on perimeter Pi. $L$ is the longest segment.

$$
H l_{i, j}=\sqrt{\left(\left(x_{i}-x_{j}\right)^{2}+\left(y_{i}-y_{j}\right)^{2}\right.}
$$

The longest Euclidean distance $W S$ between pairs of points on parameter $P i=(x i, y i)$ segment that is perpendicular to the $L$ segment. Let $W l(i, j)$ be the distance between the pairs of point on perimeter perpendicular to L segment.

$$
\begin{aligned}
& W l_{(i, j)}=\sqrt{\left(\left(x_{i}-x_{j}\right)^{2}+\left(y_{i}-y_{j}\right)^{2}\right.} \\
& W=W l_{\max }
\end{aligned}
$$

(7) (Tanabata et al., 2012) 


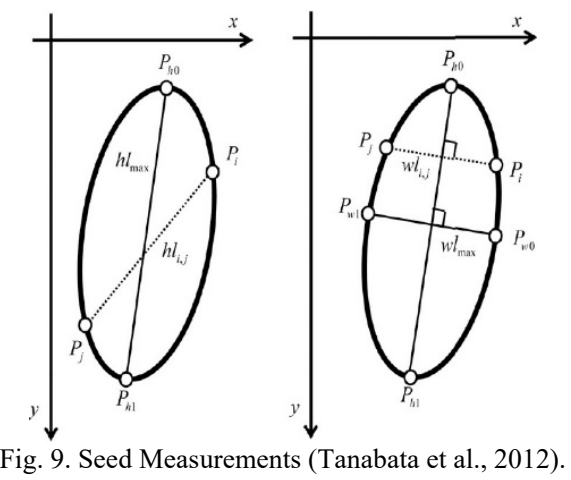

Then to extract the color and texture features, a masking operation is performed. The masking is performed on the color image $S_{l \times w}$ for the extraction of color and on gray scale image $G_{l \times w}$ for the extraction of texture features

The labeled matrix $L m$ is used to generate the mask. Let $M s_{l \times w}(c)$ be the mask where $\mathrm{c}=1$ to $\mathrm{z}$, where $\mathrm{z}$ is the total number of seeds in the image.

$$
M s_{l \times w}(c)=\left\{\begin{array}{l}
1, \text { if } \quad L m_{l \times w}=c \\
0, \quad \text { otherwise }
\end{array}\right.
$$

\subsubsection{Color Features}

For every seed the mask is created, then the mask is dot multiplied by color image $S_{l \times w(C H)}$ to obtain the color feature that are Red, Green, and Blue. Let $\operatorname{Col}_{l \times w(C H)}(c)$ be the masked color image of individual seed.

$$
\operatorname{Col}_{l \times w(C H)}(c)=\left\{\begin{array}{c}
S_{l \times w(C H=\text { red })} \times M s_{l \times w}(c) \\
S_{l \times w(C H=\text { green })} \times M s_{l \times w}(c) \\
S_{l \times w(C H=\text { blue })} \times M s_{l \times w}(c)
\end{array}\right.
$$

The average Red, Green, Blue color feature estimated. Let $R(c), G(c), B(c)$ be the color feature of individual seed in an image.

$$
\begin{aligned}
R(c) & =\frac{\sum_{1}^{m l} \sum_{1}^{w} \operatorname{Col}(l, w, C H=\text { red })(c)}{A(c)} \\
G(c) & =\frac{\sum_{1}^{m l} \sum_{1}^{w} \operatorname{Col}(l, w, C H=\text { green })(c)}{A(c)} \\
B(c) & =\frac{\sum_{1}^{m l} \sum_{1}^{w} \operatorname{Col}(l, w, C H=b l u e)(c)}{A(c)}
\end{aligned}
$$

The HSV color space of each seed in the image is computed based on (Li Jinwei et al., 2008) once R, G, and B have been achieved.

\subsubsection{Texture Features}

For extracting the texture features, the mask will be applied on the gray-scale image.

$$
G M_{l \times w}(c)=\left\{G_{l \times w} \times M s_{l \times w}(c)\right\}
$$

Four different GLCM texture features (contrast, correlation, energy, and homogeneity) are extracted at four different offsets $(0,45,90$ and 135 degree) at 256 gray levels since more levels imply more precise extraction of textural data. These features are extracted applying the equations in (Symons \& Fulcher, 1988).

Using the technique proposed in (Amadasun \& King, 1989), the NGTDM texture features have been calculated, five different features (coarseness, contrast. busyness, complexity, and texture strength). Additionally the SFTA features are computed as proposed in (Costa et al., 2012)(Costa et al., 2012). However, those SFTA features made no distinction between the various varieties. Since SFTA texture features did not discriminate well between varieties, a total of 21 texture features were chosen.

\subsection{Uniformity Estimation and New Features}

This process involves estimating uniformity based on the region of the rice seeds. The percentage of seed areas that are close to the average value is calculated. This characteristic reveals the seed size uniformity.

Second, some shape features are computed from the morphological features. 
- $\quad$ Length to width ratio

It is also known as eccentricity; it is the ratio of major axis $L$ to the minor axis $W$ of object.

$$
\mathbf{L W R}=\frac{\mathbf{L}}{\mathbf{W}}
$$

- Circularity

It relates the shape of the object with a circle and is ratio of object area to the area of the circle.

$$
\mathbf{C S}=\frac{\mathbf{4 A}}{(\mathbf{P t})\left(\mathbf{L}^{2}\right)}
$$

- Equivalent diameter

It is the diameter of the circle with the same area as the region of the rice seed.

$$
\operatorname{EQS}=\sqrt{4 \times \frac{A}{\pi}}
$$

- Compactness

It is the measure of the rice kernel roundness

$$
\cos =\frac{\sqrt{4 \times \frac{A}{\pi}}}{L}
$$

The following shape factors were derived (Symons \& Fulcher, 1988) as follow:

$$
\begin{aligned}
& \text { SF1 }=\frac{L}{A} \\
& \text { SF2 }=\frac{A}{L^{3}} \\
& \text { SF3 }=\frac{A}{\left(\frac{L}{2}\right)\left(\frac{W}{2}\right)} \\
& \text { SF4 }=\frac{A}{\left(\frac{L}{2}\right)\left(\frac{W}{2}\right) \pi}
\end{aligned}
$$

For each seed variety, 39 different features were calculated. A total of 21 textures were chosen based on their average values. The features with identical values across varieties were eliminated. We calculated the ratio of these 21 texture features to the 12 morphological features. There was a total of 252 texture to morphological ratio features found. The following are the texture features that were chosen: There are 16 GLCM features. There are also five NGTDM features.

\subsection{Development of Feature Set}

There was a total of 291 features in the final feature vector. The final feature set computed for each seed in the image is denoted by $F_{\text {seed }}$. Let $M$ represent the feature set with all 12 morphological features, $C$ represent the feature set with 6 color features, $T$ represent the feature set with 21 texture features, and $M T$ represent the feature set with 252 morphological to texture features.

$$
\boldsymbol{F}_{\text {seed }}=[\mathrm{M}, \mathrm{C}, \mathrm{T}, \mathrm{MT}]
$$

\subsection{Feature Selection for Optimal Classification}

In this phase SVM has been applied, and an indigenous feature set has been established with a classification accuracy of $95 \%$ over three different rice varieties (Giza 171, Giza 176, and Giza 181).

A total of 291 features are computed for each rice kernel from each colored image of rice seed. For classification purposes, this broad collection of features contains redundant, noisy, and irrelevant information. The classification accuracy of each feature has been computed to select the best feature set for classification, those with the greatest discriminating ability.

Then, based on the classification accuracy of features, indigenous feature sets were developed. There were 33 feature sets produced, with classification accuracy ranging from $40 \%$ to 72 percent. The accuracy of these feature sets was then investigated. For classification, the feature set with the highest accuracy was chosen.

Since the feature set is non-linear, a multiclass SVM (Pradhan et al., 2020) was used for classification using RBF (radial basis kernel function (Boser et al., 1992; Cristianini \& Shawe-Taylor, 2000)). Each variety was scanned ten times, yielding approximately 1200 kernels per variety. A total of seven images are used to train the classifier. The test images are labeled using multiclass SVM using 100 test images of rice samples, 25 samples per variety with lower resolution and less kernels.

Each feature's classification accuracy has been calculated the following figure show a sample of the calculated accuracy for some of the features. 


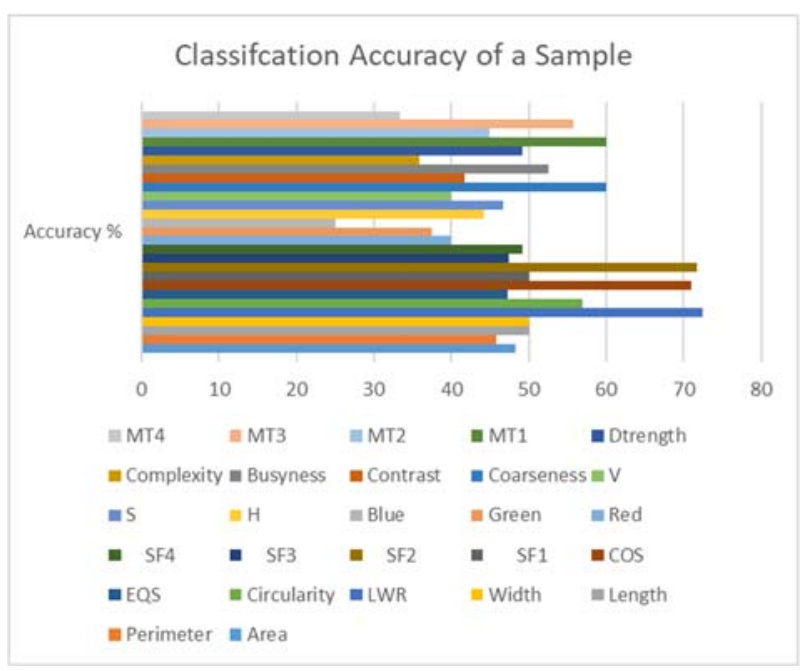

Fig. 10. Classification Accuracy for each feature

Figure 11 shows the classification accuracy of various feature sets, such as morphological, texture, NGTDM texture, GLCM texture, RGB color, and HSV color elements, among others.

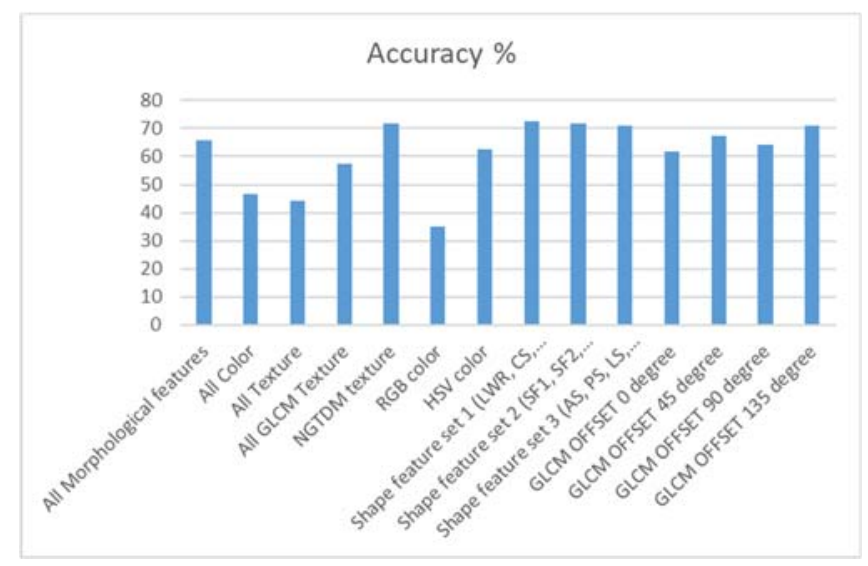

Fig. 11. Classification Accuracy of different feature sets

The classification accuracy of the feature sets and the number of features in each feature set are shown in Table 1. These feature sets are built on the basis of classification accuracy starting at $50 \%$, for example, feature set $60 \%$ contains all features with classification accuracy greater than or equal to $60 \%$. In total, 33 feature sets were developed.

\begin{tabular}{|l|c|c|c|c|c|c|c|c|c|c|c|c|c|c|c|c|c|c|c|c|c|c|}
\hline Feature Set & $\begin{array}{c}50 \\
\%\end{array}$ & $\begin{array}{c}51 \\
\%\end{array}$ & $\begin{array}{c}52 \\
\%\end{array}$ & $\begin{array}{c}53 \\
\%\end{array}$ & $\begin{array}{c}54 \\
\%\end{array}$ & $\begin{array}{c}55 \\
\%\end{array}$ & $\begin{array}{c}56 \\
\%\end{array}$ & $\begin{array}{c}57 \\
\%\end{array}$ & $\begin{array}{l}58 \\
\%\end{array}$ & $\begin{array}{c}59 \\
\%\end{array}$ & $\begin{array}{c}60 \\
\%\end{array}$ & $\begin{array}{c}61 \\
\%\end{array}$ & $\begin{array}{l}62 \\
\%\end{array}$ & $\begin{array}{c}63 \\
\%\end{array}$ & $\begin{array}{c}64 \\
\%\end{array}$ & $\begin{array}{c}65 \\
\%\end{array}$ & $\begin{array}{c}66 \\
\%\end{array}$ & $\begin{array}{c}67 \\
\%\end{array}$ & $\begin{array}{c}68 \\
\%\end{array}$ & $\begin{array}{c}69 \\
\%\end{array}$ & $\begin{array}{c}70 \\
\%\end{array}$ & $\begin{array}{c}71 \\
\%\end{array}$ \\
\hline $\begin{array}{l}\text { Number of } \\
\text { Features }\end{array}$ & 12 & 11 & 10 & 98 & 96 & 87 & 74 & 66 & 58 & 48 & 39 & 35 & 34 & 31 & 26 & 23 & 20 & 17 & 14 & 9 & 8 & 6 \\
\hline Classification & 25 & 26 & 28 & 28 & 28 & 28 & 31 & 32 & 33 & 36 & 53 & 56 & & 69 & 75 & 82 & 84 & 90 & 94 & 73 & 73 & 75 \\
Accuracy & .8 & .4 & .1 & .3 & .3 & .3 & .6 & .5 & .3 & .6 & .3 & .9 & 66 & 16 & .7 & .5 & .3 & .1 & .2 & .8 & .5 & .2 \\
\hline
\end{tabular}

Table 1. Number of Features vs Classification Accuracy.

The highest level of classification accuracy attained by a single feature was 71.5 percent. Figure 11 shows that morphological, color, and texture attributes alone do not provide the highest level of accuracy. The feature sets that were created based on the percentage accuracy of each feature indicate that feature sets with the most features have the lowest accuracy. The feature set of features with a 68 percent accuracy or higher achieved the highest accuracy. It had a $94.2 \%$ accuracy rate.

This feature set includes 14 features, the first three are LWR, COS, and SF2. While the remaining features belongs to morphological to texture. Thus, the morphological to texture ratio is very critical, as evidenced by the fact that 11 of the 14 features in the feature set are morphological to texture ratios. As a result, the added feature sets are extremely useful in classifying seeds. 


\section{Results Discussion}

Three different rice kernels have been chosen Giza 177, GIZA 178, and Sakha 101(Tantawi et al., 2004; Tantawi Badawi A., 1997). Different features of seed have been examined, and the relationship between some of these characteristics and potential yield has been investigated. Where potential yield refers to the varieties' full output capacity in tonnes per hectare. The following figures present the main characteristics for the scanned images of those varieties.

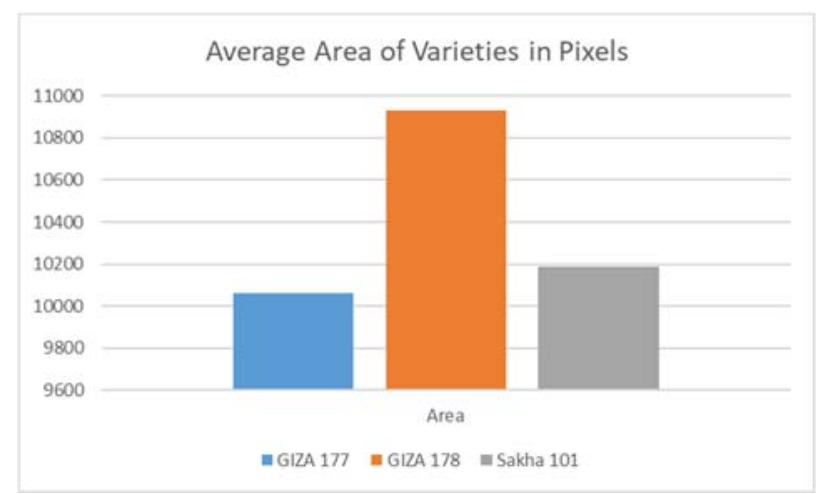

Fig. 12. Average Area of Varieties in Pixels

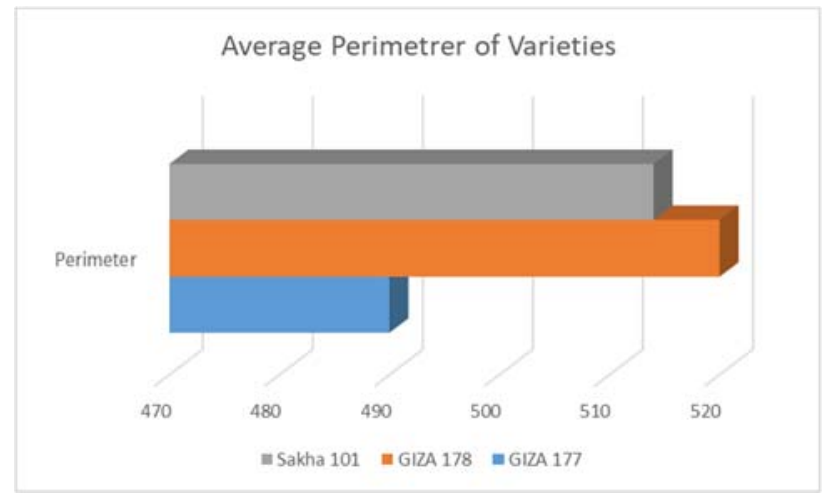

Fig. 13. Average Area of Varieties in Pixels

The seed varieties GIZA 178 and GIZA 177 have the largest and smallest average seed areas, respectively, according to the average area data. The average yield of each variety is shown in figure 14 . The data about potential yield of varieties was acquired from (Tantawi et al., 2004). The seed variety Sakha 101 has the highest yield potential.

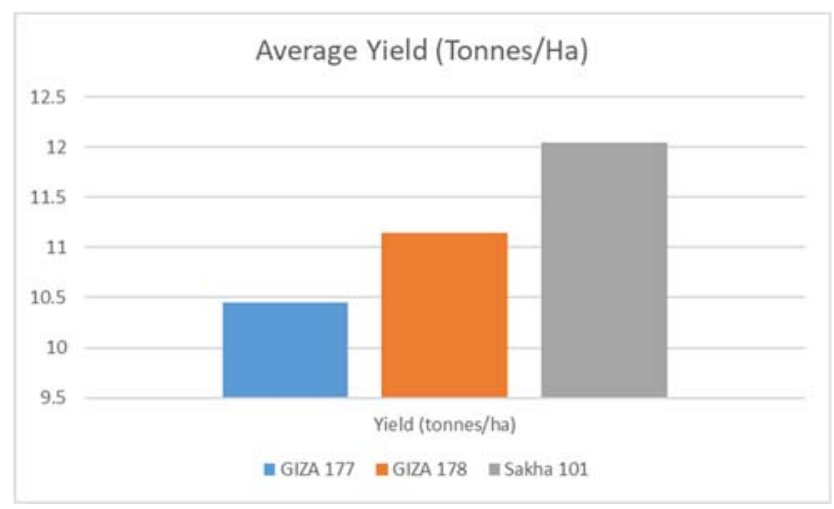

Fig. 14. Average Yield (Tonnes/Ha)

The research of seed crops is greatly aided by the study of the results obtained from image processing. Until now, several countries have estimated seed features manually using calipers and other conventional methods. The seed size of the listed varieties has been classified as medium sized seeds according to the data obtained from the Food and Agriculture Organization, despite the fact that image analysis reveals a significant difference in their size 
distribution. As a result, image analysis is a fast, simple, and accurate method for estimating seed characteristics. Many countries neglect the texture and color details on rice seeds, despite the fact that they provide critical information about crop seeds and can be extremely useful in plant research. The color feature had a classification accuracy of 62.5 percent, and the texture feature had a maximum accuracy of 71 percent, which was the same as the morphological features. As a result, these characteristics are just as important as morphological characteristics.

\section{Conclusion}

In this paper, the New features such as morphological to texture ratios has been proposed. An indigenous data set was developed, with a capable accuracy of 94.5 percent. The image acquisition technique is simple and straightforward, reducing the amount of pre-processing work needed. For each seed in an image, the NGTDM, GLCM, and SFTA texture features are computed to provide the most details about the seed's texture. The proposed algorithm can be used to research and classify any other seed form, such as wheat, corn, and so on.

The future work will be focused on identifying and exploring agricultural problems that can be solved easily and efficiently using image processing.

\section{References}

[1] Ali, A., Qadri, S., Mashwani, W. K., Brahim Belhaouari, S., Naeem, S., Rafique, S., Jamal, F., Chesneau, C., \& Anam, S. (2020). Machine learning approach for the classification of corn seed using hybrid features. International Journal of Food Properties, 23(1), 1110-1124. https://doi.org/10.1080/10942912.2020.1778724

[2] Amadasun, M., \& King, R. (1989). Textural features corresponding to textural properties. IEEE Transactions on Systems, Man, and Cybernetics, 19(5), 1264-1274. https://doi.org/10.1109/21.44046

[3] Belsare, P., \& Dewasthale, M. (2013). Application Of Image Processing For Seed Quality Assessment: A Survey. International Journal of Engineering Research and Technology, 2.

[4] Boser, B. E., Guyon, I. M., \& Vapnik, V. N. (1992). A training algorithm for optimal margin classifiers. Proceedings of the Fifth Annual Workshop on Computational Learning Theory - COLT'92, 144-152. https://doi.org/10.1145/130385.130401

[5] Chaugule, A., \& Mali, S. N. (2013). Area measurement of seed from distorted images for quality seed selection. 2013 Nirma University International Conference on Engineering (NUiCONE), 1-6. https://doi.org/10.1109/NUiCONE.2013.6780092

[6] Chaugule, M. A. (2012). Application of image processing in seed technology: A survey. 2, 153-1259.

[7] Comparison of three methods for classification of pizza topping using different color space transformations. (2005). Journal of Food Engineering, 68(3), 277-287.

[8] Costa, A. F., Humpire-Mamani, G., \& Traina, A. J. M. (2012). An Efficient Algorithm for Fractal Analysis of Textures. 2012 25th SIBGRAPI Conference on Graphics, Patterns and Images, 39-46. https://doi.org/10.1109/SIBGRAPI.2012.15

[9] Cristianini, N., \& Shawe-Taylor, J. (2000). An Introduction to Support Vector Machines and Other Kernel-based Learning Methods (1st ed.). Cambridge University Press. https://doi.org/10.1017/CBO9780511801389

[10] Fabiyi, S. D., Vu, H., Tachtatzis, C., Murray, P., Harle, D., Dao, T. K., Andonovic, I., Ren, J., \& Marshall, S. (2020). Varietal Classification of Rice Seeds Using RGB and Hyperspectral Images. IEEE Access, 8, $22493-22505$. https://doi.org/10.1109/ACCESS.2020.2969847

[11] Haralick, R. M., Shanmugam, K., \& Dinstein, I. (1973). Textural Features for Image Classification. IEEE Transactions on Systems, Man, and Cybernetics, SMC-3(6), 610-621. https://doi.org/10.1109/TSMC.1973.4309314

[12] Kaur, J. (2014). DIGITAL IMAGE ANALYSIS BASED ON AUTOMATED COUNTING CLUSTERED SOYBEAN SEEDS. INTERNATIONAL JOURNAL OF COMPUTERS \& TECHNOLOGY, 12(3), 3325-3328. https://doi.org/10.24297/ijct.v12i3.3245

[13] Kumar, M., Bora, G., \& Lin, D. (2013). Image processing technique to estimate geometric parameters and volume of selected dry beans. Journal of Food Measurement and Characterization, 7(2), 81-89. https://doi.org/10.1007/s11694-013-9142-7

[14] Li Jinwei, Liao Guiping, \& Xiao Fen. (2008). Rapeseed seeds colour recognition by machine vision. 2008 27th Chinese Control Conference, 146-149. https://doi.org/10.1109/CHICC.2008.4604918

[15] Liao, P.-S., Chen, T.-S., \& Chung, P.-C. (2001). A Fast Algorithm for Multilevel Thresholding. J. Inf. Sci. Eng., $17,713-727$.

[16] Liu, L. zhong, Zhang, W., Shu, S. bao, \& Jin, X. (2013). Image Recognition of Wheat Disease Based on RBF Support Vector Machine. Proceedings of the 2013 International Conference on Advanced Computer Science and Electronics Information. 2013 International Conference on Advanced Computer Science and Electronics Information, Beijing, China. https://doi.org/10.2991/icacsei.2013.77

[17] Mosavian, S., \& Eshraghi-Nejad, M. (2013). The effects of seed size and salinity on seed germination characteristic in wheat (var. Chamran). International Journal of Farming and Allied Sciences.

[18] Pandey, N., Krishna, S., \& Sharma, S. (2013). Automatic Seed Classification by Shape and Color Features using Machine Vision Technology. International Journal of Computer Applications Technology and Research, 2(2), 208-213. https://doi.org/10.7753/IJCATR0202.1023

[19] Pazoki, A., Farokhi, F., \& Pazoki, Z. (2013). Corn Seed Varieties Classification Based on Mixed Morphological and Color Features Using Artificial Neural Networks. Research Journal of Applied Sciences, Engineering and Technology, 6(19), 3506-3513. https://doi.org/10.19026/rjaset.6.3553

[20] Pradhan, D., Sahoo, B., Misra, B. B., \& Padhy, S. (2020). A multiclass SVM classifier with teaching learning based feature subset selection for enzyme subclass classification. Applied Soft Computing, 96, 106664. https://doi.org/10.1016/j.asoc.2020.106664

[21] Shahin, M. A., \& Symons, S. (2005). Seed sizing from images of non-singulated grain samples. Canadian Biosystems Engineering / Le Genie Des Biosystems Au Canada, 47, 3.49-3.55.

[22] Symons, S. J., \& Fulcher, R. G. (1988). Determination of wheat kernel morphological variation by digital image analysis: II. Variation in cultivars of soft white winter wheats. Journal of Cereal Science, 8(3), 219-229. https://doi.org/10.1016/S0733-5210(88)80033-X

[23] Tanabata, T., Shibaya, T., Hori, K., Ebana, K., \& Yano, M. (2012). SmartGrain: High-Throughput Phenotyping Software for Measuring Seed Shape through Image Analysis. Plant Physiology, 160(4), 1871-1880. https://doi.org/10.1104/pp.112.205120

[24] Tantawi Badawi A. (1997). Rice research accomplishments in Egypt. In Chataigner J. (Ed.), Activités de recherche sur le riz en climat méditerranéen: Vol. 24(2) (pp. 105-116). Montpellier : CIHEAM. http://om.ciheam.org/om/pdf/c24-2/CI011091.pdf 
[25] Tantawi, Badawi, A., \& Tantawi, B. (2004). Rice-based Production Systems for Food Security and Poverty Alleviation in the Near East and North Africa: New Challenges and Technological Opportunities.

[26] Truong Hoang, V., Van Hoai, D. P., Surinwarangkoon, T., Duong, H.-T., \& Meethongjan, K. (2020). A comparative study of rice variety classification based on deep learning and hand-crafted features. ECTI Transactions on Computer and Information Technology (ECTICIT), 14(1), 1-10. https://doi.org/10.37936/ecti-cit.2020141.204170

[27] Varma, V., \& Kanakadurga, K. (2013). Seed image analysis: Its applications in seed science research. 1, 30-36.

\section{Authors Profile}

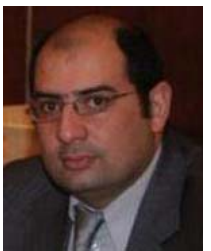

Mohammed Habib received the B.E. and M.E. degrees, from Suez Canal Univ. in 2000 and 2004, respectively. He received the Dr. Eng. degree from Suze Canal Univ. in 2009. After working as a research assistant (from 2000), an assistant professor (from 2009) in the Dept. of Electrical Engineering, Suze Canal Univ., His research interest includes Artificial Intelligence, Machine Learning, Deep Learning, Image Processing and Computer vision. 